\title{
Notiz über den Veränderlichen 136.1907 Andromedae.
}

Am 29. Januar beobachtete ich den Stern wieder im abnehmenden Licht. Um $5^{\mathrm{h}} 3^{8^{\mathrm{m}}} \mathrm{M}$. Z. Utrecht war er bereits etwa $\circ{ }_{4}^{\mathrm{m}}$ schwächer als gewöhnlich. Um halb elf hatte er das Minimum noch nicht erreicht. Die Kurve läuft derjenigen vom 25 . Dezember $x 907$ gut parallel; es geht aus sämtlichen Beobachtungen dieser beiden Abende eine Periode von $34 \stackrel{d}{9} 3$ mit genügender Sicherheit hervor. Die A.N. 4229 mitgeteilte Beobachtung von Bohlin wird durch diese Periode völlig bestätigt.

Leider war es hier am 30 . Januar trube, so da $B$ der Aufstieg verfehlt wurde. $\mathrm{Da}$ aber aus der Periode $34^{\mathrm{d}} .93$ folgt, daß der Stern am I I. September um $I 7^{\mathrm{h}} \cdot 3$ (M. Z. Gr.) das Minimum erreichte, und van Biesbroeck ihn (A. N. 42 II) am I 2. September noch um $16^{\text {h}}$. 1 schwach sah, so dauert das stationäre Minimum wenigstens 22.8 Stunden.

Utrecht, I 908 Febr. 2.
Auch nach meinen seit dem ro. November angestellten Beobachtungen haben sich die Teiler 3 und 4 der Periode als unzulässig erwiesen. Über den Teiler 2 blieb immer noch Zweifel bestehen, welcher aber durch die Notiz des Herrn Prof. Hartwig (A. N. 4229) jetzt auch beseitigt worden ist. Der Stern hätte nämlich, wie aus der Formel $^{1}$ )

$$
2417935.51+\frac{34.93}{2} E
$$

folgt, am 3. November schon lange vor dem Eintritt der Dämmerung das Minimum erreichen müssen, während Hartwig ihn um 6.4 hell sah.

Vorläufig wird nur noch der Abstieg am 4. März (bis $9^{\text {h }}$ M.Z. Gr.), der Aufstieg also am 5. März und vielleicht noch am 9. April beobachtet werden können.

1) In Nr. 4229 p. 72 Z. I 7 v. u. ist statt: 2417835.51 zu lesen: 2417935.5 I.

\section{Sternschnuppenregen am 2. Januar 1908.}

(Vergl. Astr. Nachr. $423^{\circ}$ ).

Da bezüglich auffallender Meteorschauer auch negative Angaben brauchbar sind, möchte ich mitteilen, daß ich um den 2. Januar zu folgenden Ortszeiten die helleren Veränderlichen beobachtet habe, ohne daß mir irgend welcher Reichturn an Sternschnuppen aufgefallen wäre:

$$
\begin{aligned}
& \text { Jan. } 0^{\mathrm{d}} 5^{\mathrm{h}} 33^{\mathrm{m}}-5^{\mathrm{h}} 5^{1^{\mathrm{m}}} \\
& \text { I } 539-6 \quad 18 \\
& 2 \begin{array}{llll}
2 & 24 & -6 & 17
\end{array}
\end{aligned}
$$

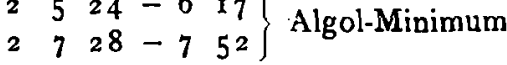

Münster, I 908 Febr. 6.

\section{A. A. Nijland.}

$$
\begin{aligned}
\text { Jan. } 3^{\mathrm{d}} 5^{\mathrm{h}} 29^{\mathrm{m}}-5^{\mathrm{h}} 59^{\mathrm{m}} \\
383^{0}-85^{2}
\end{aligned}
$$

Ein von mir notiertes Meteor Jan. $0^{\mathrm{d}} 7^{\mathrm{h}} 25^{\mathrm{m}} \circ$, Bahn $300^{\circ}+81^{\circ}$ bis $230^{\circ}+80^{\circ}\left[\varphi=+51^{\circ} 5^{\prime} ;\right.$ Red. auf Greenwich $\left.=-30^{\mathrm{m}} .5\right]$ gehört $\mathrm{zu}$ einem anderen Radianten.

In der Nähe des von den dänischen Beobachtern angegebenen Radianten $300^{\circ}+61^{\circ}$ liegt $F=297^{\circ}+53^{\circ}$; nach Heis, Resultate, S. I62, ist dieser Radiant, den Denning in $295^{\circ}+53^{\circ}$ setzt, Jan. $1-20$ tätig. Die anderen von $H$. angegebenen Radianten liegen in viel weiterem Abstande.

\section{F. Plassmann.}

\section{Trabantenphänomene Jupiters.}

Am 22. Januar I 908 fand ein dunkler Vortibergang des III. Mondes statt. Der Schatten trat aus $10^{\mathrm{h}} 39^{\mathrm{m}} 40^{\mathrm{s}}$ bis $10^{\mathrm{h}} 4^{6^{\mathrm{m}}} 2 \mathrm{O}^{\mathrm{s}}$ M. E. Z., der inzwischen auf dem matten Jupitersrande wieder hell gewordene Mond $1 \mathrm{r}^{\mathrm{h}} 23^{\mathrm{m}} 15^{\mathrm{s}}$ bis ${ }^{1} I^{h} 29^{\mathrm{m}} 30^{\mathrm{s}}$; also fanden die letzten Berührunged statt 1.7 bezw. 1.5 Minuten fruher als die Angaben des Nautical Almanac.

Am 23. Januar bedeckte der I. Trabant dea II. zentral; Landstuhl, Januar 1908. die Berührungen fanden bei unruhiger Luft statt, aber aus allen Phasen des Vorganges folgt eine zentrale Konjunktion um $12^{\mathrm{h}} 5^{\mathrm{I}^{\mathrm{m}}}$ M. E. Z.

Die Vergrößerung am I $90 \mathrm{~mm}$ Objektiv von Heyde war am 22. Jauuar mit einem älteren Huyghensokular 240. fach, am 23. Januar mit einem besseren monozentrischen Okular 223 -fach.

Uhrzeit bis auf ca. 5 Sekunden zuverlässig.

(524) Fidelio. Corr. all'effem. (V.R.I. 34): 1908 Febbrajo $6+23^{\mathrm{m}} 45^{\mathrm{s}}-2^{\circ}$ o.' I. Gr. $12^{\mathrm{m}}$ I. E. Millosevich, G. Zappa.

\footnotetext{
Inhalt zu Nr. 4233. W. Luther. Ringmikrometerbeobachtungen von kleinen Planeten. I29. - E. Schaer. Le prisme objectif employé pour l'étude du spectre solaire. 137. - F. Küistner. Bemerkungen and Berichtigungen zur Bonner Südlichen Durchmusterung. I 37 . M. Miindler. Beobachtungen des Planeten (433) Eros. 139. - E. Weiß. Fortsetzung der Ephemeride des Kometen I9o7 a. 139. - M. Ebell. Komet I907 e vor der Entdeckung. I4I. - M. Wolf. Beobachtungen des Enckeschen Kometen 1908 a und des Planeten I908 BN. I4I. - M. Wolf. Photographische Aufnahmen kleiner Planeten. I4I. - E. Millosevich. Posizione della stella AG Cbr M. I155. I41. - A. A. Nijland. Notiz über den Veränderlichen I36.rgo7 Andromedae. I43. - F. Plassmann. Sternschnuppenregen am 2. Januar 1908. 143. - Ph. Fauth. Trabantenphänomene Jupiters. 143. - E. Millosevich, G. Zappa. (524) Fidelio. 143.
} 